\title{
Therapeutic Advances for Pancreatic Cancer: a Slow and Challenging Feat that Continues to Evolve
}

\section{Carmelo J Blanquicett, MD, PhD, FACP* and Luis E. Aguirre, MD}

Departments of Hematology and Oncology, Moffitt Cancer Center, Tampa, Fl, USA

*Corresponding Author: Carmelo J. Blanquicett, MD, PhD, FACP, Departments of Hematology and Oncology, Moffitt Cancer Center, Tampa, Fl, USA.

E-mail: Carmelo.Blanquicett@moffitt.org
Received: June 05, 2021;

Published: June 23, 2021

(C) All rights are reserved by Carmelo J

Blanquicett, MD, PhD, FACP and Luis E.

Aguirre, MD.

\begin{abstract}
Despite precision medicine and advances in oncology such as immunotherapy and targeted therapy that have made substantial strides in certain solid malignancies, effective treatment options are still needed for pancreatic cancer, a highly-lethal disease with a dismal prognosis. Current treatment regimens with antimetabolites and taxanes have only modestly improved survival rates, and surgery remains the only curative measure, with just a minority of patients being eligible for curative surgery at the time of diagnosis. The role of traditional anti-cancer approaches such as radiation has yet to be definitively determined for this malignancy, and more contemporary approaches such as targeted therapy are limited in pancreatic cancer. Exploring alternate expression pathways that can be selectively targeted along with the combination of existing strategies with checkpoint inhibition or CAR-T technology, for example, may prove to be successful in making significant headway for this disease.
\end{abstract}

Keywords: Pancreatic Cancer; Immunotherapy; Targeted Therapy; CAR-T; Clinical Trials

\section{Introduction}

Survival outcomes remain poor for pancreatic cancer, the fourth leading cause of cancer related death in the Western world [1]. Despite precision medicine and advances in oncology with respect to immunotherapy and targeted therapy having made strides in other malignancies, effective treatment options are still needed for this highly lethal disease with a dismal prognosis and 5-year survival outcomes of $10 \%$ [1].

Current treatment options with antimetabolites such as gemcitabine, fluoropyrimidines, and taxanes remain the mainstay of therapy, with surgery being the only curative measure. Unfortunately, regimens such as 5-fluorouracil, oxaliplatin, irinotecan, leucovorin (FOLFIRINOX) have only modestly improved survival rates; further, patients who are considered for FOLFIRINOX treatment require good performance status. There is also the challenge in that the role of more traditional cancer treatment approaches, for example, radiation, remains to be clearly defined for this malignancy. This is the case despite years of investigation and especially so for resectable and locally advanced disease. Recently, at GI ASCO
2021, the Alliance study was presented but resulted in early closure of the treatment arm that included stereotactic body radiation therapy (SBRT) due to the perceived futility of this modality in the neoadjuvant setting, for borderline resectable pancreatic cancer patients [2].

Previously, the LAP07 trial failed to show a survival benefit with radiation [3] and thus, what can be concluded is that the role of radiation continues to evolve and a definitive benefit remains undefined, thus adding to the challenge.

\section{Small molecule inhibitors and targeted therapy}

Epidermal growth factor receptor

The epidermal growth factor receptor is upregulated in pancreatic cancer and while preclinical models suggested EGFR inhibition to be encouraging, several trials, including a phase III clinical trial as well as metanalyses reports have suggested no survival benefit but instead, added toxicities were observed by inhibiting this pathway in pancreatic cancer [4]. 
Vascular endothelial growth factor

While vascular endothelial growth factor (VEGF) has been associated with proliferation, enhanced tumor size and poor prognosis in pancreatic cancer patients, VEGF inhibition has not resulted in a meaningful benefit for this disease. Similarly, the RAS GTPases that are mutated with high frequency in pancreatic cancer [5] including KRAS, NRAS and HRAS, have been evaluated for this malignancy, with inhibition of RAS signaling thought to be a rational and promising anticancer strategy. Unfortunately, this strategy has not been proven to be an effective means to treat pancreatic cancer and most of the KRAS mutations are not targetable. Sotorasib, for example, was recently approved for G12C KRAS mutated lung cancer, but this particular mutation is only found in less than $1 \%$ of pancreatic cancer cases.

\section{Poly (ADP-Ribose) polymerase inhibitors (PARP-i)}

Pancreatic cancer infrequently results as a consequence of germline mutations (5 - 10\%) and more commonly, it is due to accumulating somatic mutations. Genetic mutations associated with pancreatic cancer include BRCA2, PALB2, BRCA1, ATM among others to name a few, with BRCA2 occurring most frequently with an incidence of less than 10\% [6]. This renders these tumors as a potential target to PARP inhibition. PARP is a family of DNA repair proteins that binds to DNA strand breaks to initiate its repair. Tumors deficient in DNA damage repair mechanisms tend to respond to platinum-based treatment, but by using PARP pathways as salvage mechanisms, these tumors can "resist" treatment and evade tumor cell death. PARP inhibitors are currently employed as maintenance monotherapy or in combination regimens, and several trials are ongoing that are evaluating the combination of these with immunotherapy (IO) or along with IO and MEK inhibition, versus targeting other DNA damage repair genes. This has been ongoing since olaparib (a PARP inhibitor) was first approved in BRCA mutated advanced pancreatic cancer, an approval that was granted as a result of the POLO trial having demonstrated a nearly 2-fold increase in PFS by using olaparib as a maintenance agent in advanced pancreatic cancer [7].

Immune therapy

Immunotherapy (IO) has revolutionized the treatment of many solid tumors, including cutaneous malignancies, while demonstrating a preferred toxicity profile as compared to standard chemotherapy regimens; unfortunately, the success of checkpoint inhibitors in pancreatic cancers has been limited. Many early phase trails failed to show a benefit of IO for this disease. Further, deficient mismatch repair (dMMR)/microsatellite instability high (MSI-H), PDL1 overexpression and high tumor mutational burden are uncommon in pancreatic cancer. Nevertheless, in patients with unresectable or metastatic cancer with $\mathrm{dMMR} / \mathrm{MSI}-\mathrm{H}$, the efficacy of pembrolizumab was reported in the Keynote 158 study with response rates in the $34 \%$ range [8]. By blocking immune checkpoints, $\mathrm{T}$ cell activation ensues that results in antitumor activity. Trials evaluating combinations with checkpoint inhibitors and chemotherapy as well as radiation are currently ongoing (ClinicalTrials.gov identifier: NCT03989310, NCT04390399) with much anticipation of the strategies that will make headway for this difficult-to-treat malignancy.

\section{Future perspectives}

As mentioned, current studies are ongoing that are evaluating the inhibition of alternate DNA repair mechanisms, investigating combinations of checkpoint inhibitors with chemotherapy, or are simply better defining the role of radiation in the borderline resectable or locally advanced setting. Connective tissue growth factor (CTGF) is overexpressed in pancreatic cancer and is involved in cell growth and metastasis, thus inhibiting CTGF is rational and has shown promise in preclinical studies [9]. It has further been suggested that inhibition of CTGF with a human monoclonal antibody may control primary and metastatic tumor growth [10]. Pamrevlumab is an antibody that inhibits CTGF. A phase III, randomized trial in patients with locally advanced, unresectable pancreatic cancer is currently recruiting to evaluate whether adding pamrevlumab to the standard gemcitabine/nab-paclitaxel or FOLFIRINOX regimens will improve survival and or downstage disease (NCT03941093). Patient reported outcomes will also be studied in this trial.

The use of chimeric antigen receptor $\mathrm{T}$ cell (CAR-T) technology in solid tumors has been limited in general. Using this technology, the patients' $\mathrm{T}$ lymphocytes are engineered to target cancer cells. CAR T cells can undergo clonal expansion in culture, to then be infused in patients with the purpose of antigen-directed, $\mathrm{T}$ cell mediated antitumor immune responses. Pre-clinical models of pancreatic cancer have found CAR $\mathrm{T}$ cell therapy platforms to be efficacious [11] and early phase clinical trials have also shown promise [12]. A phase I clinical trial of CAR-T therapy for advanced pancreatic cancer using Claudin 18.2 specific CAR-Ts, with and without nab paclitaxel showed an objective response of $82 \%$ with a 
complete response of $9 \%$ [13]. Several studies are ongoing and currently recruiting, including a trial for metastatic pancreatic cancer using autologous T cells (NCT03638193). If these are successful, it will pave the way for added therapeutic options for a disease where options are few.

\section{Conclusion}

Despite decades of investigation, breakthroughs in therapeutic advances continues to be challenging in pancreatic cancer. While numerous novel agents, immunotherapy drugs, targeted agents and strategies such as CAR-T therapy are evolving and paving the long road leading towards improvement in survival outcomes, effective treatment is still a much-needed priority for pancreatic cancer, and breakthroughs at a faster pace are essential to alter the expectation for this malignancy to become the number 2 cause for cancer deaths by 2030 [14]. In combination with precision medicine and novel cell therapy/molecular pathology elucidation, along with effective screening methods, it is hoped that what is to come will accelerate the pace towards significantly-improved outcomes for this lethal disease.

\section{Conflict of Interest Statement}

Dr. Blanquicett is an editorial board member of Acta Scientific Gastrointestinal Disorders.

\section{Bibliography}

1. https://seer.cancer.gov/statfacts/html/pancreas.html

2. Journal of Clinical Oncology 39.3 (2021): 377. DOI: 10.1200/ JC0.2021.39.3_suppl.377.

3. Hammel Pascal., et al. "Effect of Chemoradiotherapy vs Chemotherapy on Survival in Patients with Locally Advanced Pancreatic Cancer Controlled After 4 Months of Gemcitabine with or without Erlotinib. The LAP07 Randomized Clinical Trial". The Journal of the American Medical Association 315 (2016): 1844-1853.

4. Forster Tobias., et al. "Cetuximab in Pancreatic Cancer Therapy: A Systematic Review and Meta-Analysis". Oncology 98 (2020): 53-60.

5. Bryant Kristen., et al. "KRAS: Feeding Pancreatic Cancer Proliferation". Trends in Biochemical Sciences 39 (2014): 91-100.
6. Wong Winston., et al. "BRCA Mutations in Pancreas Cancer: Spectrum, Current Management, Challenges and Future Prospects". Cancer Management and Research 12 (2020): 27312742.

7. Golan Talia., et al. "Maintenance Olaparib for Germline BRCAMutated Metastatic Pancreatic Cancer". The New England Journal of Medicine 381 (2019): 317-327.

8. Marabelle Aurelien., et al. "Efficacy of Pembrolizumab in Patients with Noncolorectal High Microsatellite Instability/ Mismatch Repair-Deficient Cancer: Results From the Phase II KEYNOTE-158 Study". Journal of Clinical Oncology 38 (2020): $1-10$.

9. Albrecht Neese., et al. "CTGF antagonism with mAb FG-3019 enhances chemotherapy response without increasing drug delivery in murine ductal pancreas cancer". Proceedings of the National Academy of Sciences of the United States of America 110 (2013): 12325-12330.

10. Dornhofer Nadja., et al. "Connective tissue growth factor-specific monoclonal antibody therapy inhibits pancreatic tumor growth and metastasis". Cancer Research 66 (2006): 58165827.

11. Akce Mehmet., et al. "The Potential of CAR T Cell Therapy in Pancreatic Cancer". Frontiers in Immunology 9 (2018): 2166.

12. Siddiqui Raheel and Sardar Muhammad. "A Systematic Review of the Role of Chimeric Antigen Receptor T (CAR-T) Cell Therapy in the Treatment of Solid Tumors". Cureus 13 (2021): e14494.

13. Zhan Xianbao., et al. "Phase I trial of Claudin 18.2-specific Chimeric Antigen Receptor T cells for Advanced Gastric and Pancreatic Adenocarcinoma". Journal of Clinical Oncology 37 (2019): 2509-2509.

14. Rahib Lola., et al. "Projecting cancer incidence and deaths to 2030: the unexpected burden of thyroid, liver, and pancreas cancers in the United States". Cancer Research 74 (2014): 2913-2921.

\section{Volume 4 Issue 7 July 2021}

(C) All rights are reserved by Carmelo J Blanquicett, MD, PhD, FACP and Luis E. Aguirre, MD. 